\title{
474562 - BETA BLOCKERS INCREASE PERIOPERATIVE RISK IN ACUTE ANEMIA SURGERY
}

\author{
Scott Beattie, MD ${ }^{1}$, Keyvan Karkouti, MD ${ }^{1}$, Nicholas Mitsakakis, M.Sc. ${ }^{1}$, Duminda \\ Wijeysundera, MD FRCPC ${ }^{1}$, Gregory Hare, MD, PhD $^{2}$, Gordon Tait, PhD $^{1}$ \\ 1. Anesthesia, Toronto General Hospital, Toronto, ON, Canada \\ 2. Anesthesia, St. Michael's Hospital, Toronto, ON, Canada
}

Introduction: Mortality after non-cardiac surgery approaches $2 \%$ in many observational databases and has remained unchanged in the last decade. Cardiac events are the single largest cause of mortality. The AHA/ACC has recommended perioperative beta blockade to reduce cardiac related mortality. The recently completed POISE trial found that beta blockade increased mortality in moderate to high risk patients and also found that this was associated with perioperative transfusion. The present study was designed to evaluate the effects of beta blockers on patients who became acutely anemic in the perioperative period.

Methods: This is a retrospective analysis of 4492 consecutive patients having noncardiac, non-transplant surgery in an academic institution between 2005 and 2006. Important preoperative morbidity, drug history, details of hematological tests and post operative utilization of blood products were collected. The composite outcome of interest combined death at 90 days and perioperative myocardial infarction (defined by a troponin I elevation $>0.7 \mu \mathrm{g} / \mathrm{L}$ ). The relationship between the administration of beta blockers, the acute change in hemoglobin (preoperative - nadir postoperative) and the composite outcome was analyzed using logistic regression. Propensity scores were derived for patients administered beta blockers and a matched cohort was selected of patients who were not administered beta blockers. We then assessed the relationship between the percent change in hemoglobin and the composite outcome in each group using a cubic spine function. Statistical analysis was performed using SAS version 9.1

Results: Multivariable logistic regression analysis found that both beta blockers and acute peri-operative anemia were independent predictors of the composite outcome death and MI. The adjusted odds ratio for beta blockers was 1.8 (95\% CI 1.4 to 2.2). We also found that every 10\% decrease in hemoglobin increased the odds of death an d MI 1.33 (1.19 to 1.9) The model was accurate with an ROC $=0.88$ and well calibrated (Hosmer Lemenshow $\mathrm{p}=0.93$ ). Propensity scores were used to match 707 patients administered beta-blockers to patients with equal risk who were not administered beta blockers. In this subset of the study population, the odds ratio of the composite outcome was 1.7 (95\% CI $0.97-2.4)$. We found that the probability of mortality or myocardial infarction was progressively higher for patients administered beta blockers when hemoglobin decreased more than 30\%. Cubic spine transformations for 1414 propensity matched patients administered beta blockers and not administered beta blockers showed that the risk of the composite outcome is equal until a hemoglobin change of $30 \%$. For perioperative decreases of hemoglobin greater than $30 \%$, the administration of beta blockers progressively increases the probability of the composite outcome.

Discussion: The probability of mortality or MI increases in beta blocked patients when 
there is a perioperative fall in hemoglobin level of more than $30 \%$. This suggests that the suppression of the beta adrenergic cardiac response to acute anemia increases the risk of MI and death

References: None 